ANALYSIS \& PDE Volume 9 No. $4 \quad 2016$ L W. JIUYIZHU

INTERIOR NODAL SETS OF STEKLOV EIGENFUNCTIONS ON SURFACES 


\title{
INTERIOR NODAL SETS OF STEKLOV EIGENFUNCTIONS ON SURFACES
}

\author{
JIUYI ZHU
}

\begin{abstract}
We investigate the interior nodal sets $\mathcal{N}_{\lambda}$ of Steklov eigenfunctions on connected and compact surfaces with boundary. The optimal vanishing order of Steklov eigenfunctions is shown be $C \lambda$. The singular sets $\mathscr{S}_{\lambda}$ consist of finitely many points on the nodal sets. We are able to prove that the Hausdorff measure $H^{0}\left(\mathscr{S}_{\lambda}\right)$ is at most $C \lambda^{2}$. Furthermore, we obtain an upper bound for the measure of interior nodal sets, $H^{1}\left(\mathcal{N}_{\lambda}\right) \leq C \lambda^{3 / 2}$. Here the positive constants $C$ depend only on the surfaces.
\end{abstract}

\section{Introduction}

Let $(\mathcal{M}, g)$ be a smooth, connected and compact surface with smooth boundary $\partial \mathcal{M}$. The main goal of this paper is to obtain an upper bound of interior nodal sets

$$
\mathcal{N}_{\lambda}=\left\{z \in M \mid e_{\lambda}=0\right\}
$$

for Steklov eigenfunctions, which satisfy

$$
\begin{cases}\triangle_{g} e_{\lambda}=0, & z \in \mathcal{M}, \\ \partial e_{\lambda}(z) / \partial \nu=\lambda e_{\lambda}(z), & z \in \partial \mathcal{M},\end{cases}
$$

where $v$ is a unit outward normal on $\partial \mathcal{M}$. The Steklov eigenfunctions were introduced by Steklov in 1902 for bounded domains in the plane. They interpret the steady state temperature distribution in domains where the heat flux on the boundary is proportional to the temperature. They also have applications in quite a few physical fields, such as fluid mechanics, electromagnetism and elasticity. In particular, the model (1-1) was studied by Calderón [1980] as solutions can be regarded as eigenfunctions of the Dirichlet-to-Neumann map. The interior nodal sets of Steklov eigenfunctions represent the stationary points in $M$. In the context of quantum mechanics, nodal sets are the sets where a free particle is least likely to be found.

It is well known that the spectrum $\lambda_{j}$ of the Steklov eigenvalue problem is discrete with

$$
0=\lambda_{0}<\lambda_{1} \leq \lambda_{2} \leq \lambda_{3} \leq \cdots \quad \text { and } \quad \lim _{j \rightarrow \infty} \lambda_{j}=\infty
$$

There exists an orthonormal basis $\left\{e_{\lambda_{j}}\right\}$ of eigenfunctions such that

$$
e_{\lambda_{j}} \in C^{\infty}(\mu) \text { and } \int_{\partial \mathcal{M}} e_{\lambda_{j}} e_{\lambda_{k}} d V_{g}=\delta_{j}^{k} .
$$

This research is partially supported by the NSF grant DMS 1500468 .

MSC2010: 35P15, 35P20, 58C40, 28A78.

Keywords: nodal sets, upper bound, Steklov eigenfunctions. 
Estimating the Hausdorff measure of nodal sets has always been an important subject concerning the study of eigenfunctions. This subject centers around the famous Yau conjecture. Recently, much work has been devoted to the bounds of nodal sets

$$
Z_{\lambda}=\left\{z \in \partial \mathcal{M} \mid e_{\lambda}(z)=0\right\}
$$

of Steklov eigenfunctions on the boundary. Bellová and Lin [2015] proved $H^{m-1}\left(Z_{\lambda}\right) \leq C \lambda^{6}$ with $C$ depending only on $\mathcal{M}$ if $\mathcal{M}$ is an $m+1$-dimensional analytic manifold. Zelditch [2014] improved their results and gave the optimal upper bound $H^{m-1}\left(Z_{\lambda}\right) \leq C \lambda$ for analytic manifolds using microlocal analysis. For the smooth manifold $\mathcal{M}$, Wang and Zhu [2015] recently established a lower bound

$$
H^{m-1}\left(Z_{\lambda}\right) \geq C \lambda^{(3-m) / 2} .
$$

Before presenting our results for interior nodal sets, let's briefly review the literature about the nodal sets of classical eigenfunctions. The interested reader may refer to the book [Han and Lin 2008] and survey [Zelditch 2008] for detailed accounts about this subject. Let $e_{\lambda}$ be $L^{2}$ normalized eigenfunctions of the Laplace-Beltrami operator on compact manifolds $(\mathcal{M}, g)$ without boundary,

$$
-\triangle_{g} e_{\lambda}=\lambda^{2} e_{\lambda}
$$

Yau's conjecture states that, for any smooth manifolds, one should control the upper and lower bounds of nodal sets of classical eigenfunctions as

$$
c \lambda \leq H^{n-1}\left(\mathcal{N}_{\lambda}\right) \leq C \lambda,
$$

where $C$ and $c$ depend only on the manifold $\mathcal{M}$. The conjecture is only verified for real analytic manifolds, by Donnelly and Fefferman [1988]. Lin [1991] also showed the upper bound for analytic manifolds by a different approach. For smooth manifolds, the conjecture is still not settled. For the lower bound of nodal sets with $n \geq 3$, Colding and Minicozzi [2011] and Sogge and Zelditch [2011;2012] independently obtained that

$$
H^{n-1}\left(\mathcal{N}_{\lambda}\right) \geq C \lambda^{(3-n) / 2}
$$

for smooth manifolds. See also [Hezari and Sogge 2012] for deriving the same bound by adapting the idea in [Sogge and Zelditch 2011]. For the upper bound, Hardt and Simon [1989] gave an exponential upper bound

$$
H^{n-1}\left(\mathcal{N}_{\lambda}\right) \leq C e^{\lambda \ln \lambda} .
$$

In surfaces, better results have been obtained. Brüning [1978] and Yau (unpublished) derived the same lower bound as (1-3). The best estimate to date for the upper bound is

$$
H^{1}\left(\mathcal{N}_{\lambda}\right) \leq C \lambda^{3 / 2}
$$

by Donnelly and Fefferman [1990a] and Dong [1992] using different methods.

Let us return to the Steklov eigenvalue problem (1-1). By the maximum principle, there exist nodal sets in the manifold $\mathcal{M}$ and those sets must intersect the boundary $\partial \mathcal{M}$. Thus it is natural to study the size 
of interior nodal sets in $\mu$. We can also ask Yau-type questions about the Hausdorff measure of nodal sets. The natural and corresponding conjecture for Steklov eigenfunctions should be exactly the same as (1-3). See also the open questions in the survey by Girouard and Polterovich [2014]. Recently, Sogge, Wang and the author [Sogge et al. 2015] obtained a lower bound for interior nodal sets

$$
H^{n-1}\left(\mathcal{N}_{\lambda}\right) \geq C \lambda^{(2-n) / 2}
$$

for $n$-dimensional manifolds $\mathcal{M}$. Very recently, Polterovich, Sher and Toth [Polterovich et al. 2015] verified the Yau-type conjecture for (1-1) on real analytic Riemannian surfaces.

An interesting topic related to the measure of nodal sets is about doubling inequalities. Based on doubling inequalities, one can obtain the vanishing order of eigenfunctions, which characterizes how fast the eigenfunctions vanish. For the classical eigenfunctions of (1-2), Donnelly and Fefferman [1988; 1990b] obtained that the maximal vanishing order of $e_{\lambda}$ is of order at most $C \lambda$ everywhere. To achieve it, a doubling inequality

$$
\int_{\mathbb{B}\left(z_{0}, 2 r\right)} e_{\lambda}^{2} \leq C e^{\lambda} \int_{\mathbb{B}\left(z_{0}, r\right)} e_{\lambda}^{2}
$$

is derived using Carleman estimates, where $\mathbb{B}(p, c)$ denotes a ball centered at $p$ with radius $c$. The doubling estimate (1-4) plays an important role in obtaining the bounds of nodal sets for analytic manifolds in [Donnelly and Fefferman 1988] and the upper bound of nodal sets for smooth surfaces in [Donnelly and Fefferman 1990a]. For the Steklov eigenfunctions, we obtain a doubling inequality on the boundary $\partial \mu$ and derive that the sharp vanishing order is less than $C \lambda$ on the boundary $\partial \mathcal{M}$. For Steklov eigenfunctions in $M$, we are also able to get the doubling inequality; see Proposition 5. With the aid of doubling estimates and Carleman inequalities, the following optimal vanishing order for Steklov eigenfunctions can be obtained:

Theorem 1. The vanishing order of the Steklov eigenfunction $e_{\lambda}$ of (1-1) in $M$ is everywhere less than $C \lambda$.

Its sharpness can be seen in the case that the manifold $M$ is a ball. Notice that the doubling estimates in Proposition 5 and the vanishing order in Theorem 1 hold for any $n$-dimensional compact manifolds.

Singular sets

$$
\mathscr{S}_{\lambda}=\left\{z \in M \mid e_{\lambda}=0, \nabla e_{\lambda}=0\right\}
$$

are contained in nodal sets. In Riemannian surfaces, those singular sets consist of finitely many points in the 1-dimensional nodal sets. It is interesting to count the number of those singular sets. Based on a Carleman inequality with singularities, we are able to show an upper bound of singular sets.

Theorem 2. Let $(\mathcal{M}, g)$ be a smooth, connected and compact surface with smooth boundary $\partial \mathcal{M}$. Then

$$
H^{0}\left(\mathscr{Y}_{\lambda}\right) \leq C \lambda^{2}
$$

holds for Steklov eigenfunctions in (1-1).

For the nodal sets of Steklov eigenfunctions, we are able to build a similar type of Carleman inequality as [Donnelly and Fefferman 1990a], and show the following result: 
Theorem 3. Let $(\mathcal{M}, g)$ be a smooth, connected and compact surface with smooth boundary $\partial \mathcal{M}$. Then

$$
H^{1}\left(\mathcal{N}_{\lambda}\right) \leq C \lambda^{3 / 2}
$$

holds for Steklov eigenfunctions in (1-1).

The outline of the paper is as follows. Section 2 is devoted to reducing the Steklov eigenvalue problem into an equivalent elliptic equation without boundary. Then we obtain the optimal doubling inequality and show Theorem 1. In Section 3, we establish the Carleman inequality with singularities at finitely many points. Under additional assumptions on those singular points, a stronger Carleman inequality is derived. We measure the singular sets in Section 4. Sections 5, 6 and 7 are devoted to obtaining the nodal length of Steklov eigenfunctions. Under the condition of slow growth of $L^{2}$ norm, we find out the nodal length in Section 6. Based on a similar type of Calderón and Zygmund decomposition procedure, we show the slow growth at almost every point. Then the measure of nodal sets is derived by summing up the nodal length in each small square. The letters $c, C, C_{i}, d_{i}$ denote generic positive constants and do not depend on $\lambda$. They may vary in different lines and sections.

\section{Vanishing order of Steklov eigenfunctions}

In this section, we will reduce the Steklov eigenvalue problem to an equivalent model on a boundaryless manifold. The presence of eigenvalues on the boundary $\partial \mu$ will be reflected in the coefficient functions of a second-order elliptic equation. Let $d(z)=\operatorname{dist}\{z, \partial \mathcal{M}\}$ denote the geodesic distance function from $x \in \mathcal{M}$ to the boundary $\partial \mathcal{M}$. Since $\mathcal{M}$ is smooth, there exists a $\rho$-neighborhood of $\partial \mathcal{M}$ in $\mathcal{M}$ such that $d(x)$ is smooth in the neighborhood. Let's denote it as $\mu_{\rho}$. We extend $d(z)$ smoothly in $\mu$ by

$$
\delta(z)= \begin{cases}d(z), & z \in \mathcal{M}_{\rho}, \\ l(z), & z \in \mathcal{M} \backslash \mathcal{M}_{\rho},\end{cases}
$$

where $l(z)$ is a smooth function in $\mathcal{M} \backslash \mathcal{M}_{\rho}$. Note that the extended function $\delta(z)$ is a smooth function in $M$. We first reduce the Steklov eigenvalue problem into an elliptic equation with Neumann boundary condition. Let

$$
v(z)=e_{\lambda} \exp \{\lambda \delta(z)\}
$$

It is known that $v(z)=e_{\lambda}(z)$ on $\partial \mathcal{M}$. For $z \in \partial \mathcal{M}$, we have $\nabla_{g} \delta(z)=-v(z)$. Recall that $v(z)$ is the unit outer normal on $z \in \partial \mathcal{M}$. We can check that the new function $v(z)$ satisfies

$$
\begin{cases}\triangle_{g} v+b(z) \cdot \nabla_{g} v+q(z) v=0 & \text { in } \mathcal{M} \\ \partial v / \partial v=0 & \text { on } \partial \mathcal{M}\end{cases}
$$

with

$$
\left\{\begin{array}{l}
b(z)=-2 \lambda \nabla_{g} \delta(z) \\
q(z)=\lambda^{2}\left|\nabla_{g} \delta(z)\right|^{2}-\lambda \triangle_{g} \delta(z)
\end{array}\right.
$$

In order to get rid of the boundary condition, we attach two copies of $M$ along the boundary and consider the double manifold $\bar{M}=\mu \cup M$. The metric $g$ extends to $\bar{M}$ with Lipschitz-type singularity along $\partial \mathcal{M}$, since the lift metric $g^{\prime}$ of $g$ on $\mu$ to the double manifold $\bar{M}$ is Lipschitz. There also exists a canonical 
involutive isometry $\mathscr{F}: \bar{M} \rightarrow \bar{M}$ that interchanges the two copies of $\mathcal{M}$. Then the function $v(x)$ can be extended to $\bar{M}$ by $v \circ \mathscr{F}=v$. Therefore, $v(z)$ satisfies

$$
\triangle_{g^{\prime}} v+\bar{b}(z) \cdot \nabla_{g^{\prime}} v+\bar{q}(z) v=0 \quad \text { in } \bar{M} .
$$

From (2-3), one can see that

$$
\left\{\begin{array}{l}
\|\bar{b}\|_{W^{1, \infty}(\overline{\mathcal{M}})} \leq C \lambda \\
\|\bar{q}\|_{W^{1, \infty}(\overline{\mathcal{M}})} \leq C \lambda^{2}
\end{array}\right.
$$

After this procedure, we can instead study the nodal sets for the second-order elliptic equation (2-4) with assumption (2-5). Note that $\bar{M}$ is a manifold without boundary.

We present a brief proof of Theorem 1. It is a small modification of the argument in [Zhu 2015], where the sharp vanishing order of Steklov eigenfunctions on the boundary $\partial \mathcal{M}$ is shown to be less than $C \lambda$. To achieve it, we derive the double inequality in a neighborhood of the boundary by quantitative Carleman estimates.

Proof of Theorem 1. Recall the strategy in [Zhu 2015]; we do an even reflection in a small neighborhood of the boundary. Then we deal with a second-order elliptic equation with a Lipschitz-continuous leading coefficient function and satisfying the same conditions as (2-5). By the regularity argument for dealing with a Lipschitz metric in [Donnelly and Fefferman 1990b], the same Carleman estimates in [Zhu 2015] hold for (2-4). Let $r(z)$ be the distance function from $z$ to the fixed point $z_{0}$. If $u \in C_{0}^{\infty}\left(\mathbb{B}_{r_{0}}\left(z_{0}\right) \backslash\left\{z_{0}\right\}\right)$ and $\tau>C_{1}\left(1+\|\bar{b}\|_{W^{1, \infty}}+\|\bar{q}\|_{W^{1, \infty}}^{1 / 2}\right)$, following the arguments in [Zhu 2015] and choosing the test function $\tilde{\phi}(z)=\ln r(z)-r^{\epsilon}(z)$ there instead, we have the Carleman inequality

$$
C\left\|r^{2} e^{\tau \phi(r)}\left(\triangle_{g^{\prime}} u+\bar{b} \cdot \nabla_{g^{\prime}} u+\bar{q} u\right)\right\|_{L^{2}} \geq \tau^{3 / 2}\left\|r^{\epsilon / 2} e^{\tau \phi(r)} u\right\|_{L^{2}}+\tau^{1 / 2}\left\|r^{1+\epsilon / 2} e^{\tau \phi(r)} \nabla u\right\|_{L^{2}},
$$

where $\phi(r)=-\ln r(z)+r^{\epsilon}(z)$. See also, e.g., [Bakri and Casteras 2014] for similar estimates on manifolds with smooth metric. In particular, we have the following lemma:

Lemma 4. Let $u \in C_{0}^{\infty}\left(\frac{1}{2} \epsilon_{1}<r<\epsilon_{0}\right)$. If $\tau>C_{1}\left(1+\|\bar{b}\|_{W^{1, \infty}}+\|\bar{q}\|_{W^{1, \infty}}^{1 / 2}\right)$. Then

$$
\int r^{4} e^{2 \tau \phi(r)}\left|\triangle_{g^{\prime}} u+\bar{b} \cdot \nabla_{g^{\prime}} u+\bar{q} u\right|^{2} d r d \omega \geq C_{2} \tau^{3} \int r^{\epsilon} e^{2 \tau \phi(r)} u^{2} d r d \omega,
$$

where $\phi(r)=-\ln r(z)+r^{\epsilon}(z)$ and $0<\epsilon_{0}, \epsilon_{1}, \epsilon<1$ are some fixed constants. Moreover, $(r, \omega)$ are the standard polar coordinates.

Using this Carleman estimate and choosing suitable test functions, a Hadamard three-ball result can be obtained in $\bar{M}$ following the arguments in [Zhu 2015]. There exist constants $r_{0}, C$ and $0<\gamma<1$ depending only on $\bar{M}$ such that, for any solutions of (2-4), $0<r<r_{0}$ and $z_{0} \in \bar{M}$, one has

$$
\int_{\mathbb{B}\left(z_{0}, r\right)} v^{2} \leq e^{C\left(1+\|\bar{b}\|_{W^{1, \infty}}+\|\bar{q}\|_{W^{1}, \infty}^{1 / 2}\right)}\left(\int_{\mathbb{B}\left(z_{0}, 2 r\right)} v^{2}\right)^{1-\gamma}\left(\int_{\mathbb{B}\left(z_{0}, r / 2\right)} v^{2}\right)^{\gamma} .
$$

Based on a propagation of smallness argument using the three-ball result and Carleman estimates (2-6), as that in [Zhu 2015], taking the assumptions (2-5) into account, we are able to obtain the doubling inequality in $\bar{M}$. 
Proposition 5. There exist constants $r_{0}$ and $C$ depending only on $\bar{M}$ such that, for any $0<r<r_{0}$ and $z_{0} \in \bar{M}$,

$$
\|v\|_{L^{2}\left(\mathbb{B}\left(z_{0}, 2 r\right)\right)} \leq e^{C \lambda}\|v\|_{L^{2}\left(\mathbb{B}\left(z_{0}, r\right)\right)}
$$

for any solutions of (2-4).

One can see that the doubling estimate holds in $M$ if $\mathbb{B}\left(z_{0}, 2 r\right) \subset \mathcal{M}$. By standard elliptic estimates, one can have the $L^{\infty}$ norm doubling inequality

$$
\|v\|_{L^{\infty}\left(\mathbb{B}\left(z_{0}, 2 r\right)\right)} \leq e^{C \lambda}\|v\|_{L^{\infty}\left(\mathbb{B}\left(z_{0}, r\right)\right)} .
$$

Since $\bar{M}$ is compact, we can derive that

$$
\|v\|_{L^{\infty}\left(\mathbb{B}\left(z_{0}, r\right)\right)} \geq r^{C \lambda}
$$

for any $z_{0} \in \overline{\mathcal{M}}$, which implies the vanishing order for $v$ is less than $C \lambda$. So is the vanishing order of $u$. This completes Theorem 1.

\section{Carleman estimates}

This section is devoted to establishing Carleman inequalities involving weighted functions at finitely many points. From this section on, $\bar{M}$ is a compact Riemannian surface. We construct suitable conformal coordinate charts near $\partial \mathcal{M} \subset \bar{M}$ following the arguments in [Donnelly and Fefferman 1990a, p. 342-343], where the same construction is established for a Lipschitz double manifold. By the Riemann mapping theory in [Jost 1984], we first construct charts around $\partial \mathcal{M} \subset \mathcal{M}$. We map a half disk centered on the $x$-axis in the $(x, y)$-plane into the manifold $\mu$ with the $x$-axis mapped to $\partial \mu$. Thus, the metric is locally given as $\bar{g}(x, y)\left(d x^{2}+d y^{2}\right)$ with $y>0$. The differentiable structure and the definition of the metric on the double manifold $\bar{M}$ correspond to reflection about the $x$-axis. Thus, we have the required the conformal charts with $\bar{g}(x,|y|)\left(d x^{2}+d y^{2}\right)$ on the double manifold $\bar{M}$. Then we will consider the behavior of $v$ in a conformal coordinate patch. There exists a finite number $N$ of conformal charts $\left(U_{i}, \phi_{i}\right)$ with $\phi_{i}: U_{i} \subset \bar{M} \rightarrow \mathscr{V}_{i} \subset \mathbb{R}^{2}$ and $i \in\{1,2, \ldots, N\}$. On each of these charts, the metric is conformally flat and there exists a positive function $g_{i}$ such that $g^{\prime}=g_{i}(x, y)\left(d x^{2}+d y^{2}\right)$. By the compactness of the surface, there are positive constants $c$ and $C$ such that $0<c<g_{i}<C$ for each $i$. Under this equivalent metric, $\triangle_{g^{\prime}}=g_{i}^{-1} \triangle$, where $\triangle$ is the Euclidean Laplacian. Hence, (2-4) can be written as

$$
\Delta v+\bar{b}(z) \cdot \nabla v+\bar{q}(z) v=0 \quad \text { in } \mathscr{V}_{i},
$$

where $\nabla$ is the Euclidean gradient and $z=(x, y)$. We use the same notations $\bar{b}(z)$ and $\bar{q}(z)$ as in (3-1), since they satisfy the same conditions as (2-5). They only differ by some function about $g_{i}$.

By restricting to a small ball $\mathbb{B}(p, 3 c)$ contained in the conformal chart, we consider $v$ in the small ball. Let $\bar{v}(z)=v(c z)$. It follows from (3-1) that

$$
\triangle \bar{v}+\tilde{b}(z) \cdot \nabla \bar{v}+\tilde{q}(z) \bar{v}=0 \quad \text { in } \mathbb{B}_{3},
$$

with $\tilde{b}=c \bar{b}$ and $\tilde{q}=c^{2} \bar{q}$. If $c$ is sufficiently small, $\tilde{b}$ and $\tilde{q}$ are arbitrarily small. 
The crucial tool in [Donnelly and Fefferman 1990a] is a Carleman inequality for classical eigenfunctions involving weighted functions with singularities at finitely many points. We will obtain the corresponding Carleman inequality for the second-order elliptic equation (3-2). We adapt the approach in [Donnelly and Fefferman 1990a] to obtain the desirable Carleman estimate for (3-2).

Let $\mathscr{D} \subset \mathscr{C}$ be an open set and $\psi \in C_{0}^{\infty}(\mathscr{D})$ be a real-valued function. We introduce the differential operators

$$
\partial=\frac{1}{2}\left(\frac{\partial}{\partial x}-i \frac{\partial}{\partial y}\right) \quad \text { and } \quad \bar{\partial}=\frac{1}{2}\left(\frac{\partial}{\partial x}+i \frac{\partial}{\partial y}\right) .
$$

Direct computation shows that $\bar{\partial} \partial \psi=\frac{1}{4} \Delta \psi$. By the Cauchy-Riemann equation, $u$ is holomorphic if and only if $\bar{\partial} u=0$. For completeness, we present the elementary inequality in [Donnelly and Fefferman 1990a].

Lemma 6. Let $\Phi$ be a smooth positive function in $\mathscr{D}$. Then

$$
\int_{\mathscr{D}}|\bar{\partial} u|^{2} \Phi \geq \frac{1}{4} \int_{\mathscr{D}}(\triangle \ln \Phi)|u|^{2} \Phi .
$$

Here the integral is taken with respect to the Lebesgue measure.

We want the weight function to involve those singular points. To specialize the choice of $\Phi$, we construct the following function $\psi_{0}$ :

Lemma 7. There exists a smooth function $\psi_{0}$ defined for $|z|>1-2 a$ satisfying the following properties:

(i) $a_{1} \leq \psi_{0}(z) \leq a_{2}$ with constants $a_{1}, a_{2}>0$.

(ii) $\psi_{0}=1$ on $\{|z|>1\}$.

(iii) $\triangle \ln \psi_{0} \geq 0$ on $\{|z|>(1-2 a)\}$.

(iv) If $1-2 a<|z|<1-a$, then $\triangle \ln \psi_{0} \geq a_{3}>0$.

The existence of such a $\psi_{0}$ follows from existence and uniqueness theory of ordinary differential equations.

We assume that

$$
D_{l}=\left\{z|| z-z_{l} \mid \leq \delta\right\}
$$

Let $D_{l}$ be a finite collection of pairwise disjoint disks that are contained in a unit disk centered at the origin. Let

$$
D_{l}(a)=\left\{z|| z-z_{l} \mid \leq(1-2 a) \delta\right\}
$$

be the smaller concentric disk. We define a smooth weight function $\Psi_{0}(z)$ as

$$
\Psi_{0}(z)= \begin{cases}1 & \text { if } z \notin \bigcup_{l} D_{l}, \\ \psi_{0}\left(\left(z-z_{l}\right) / \delta\right) & \text { if } z \in D_{l} .\end{cases}
$$

We also introduce the domain

$$
A_{l}=\left\{(1-2 a) \delta \leq\left|z-z_{l}\right| \leq(1-a) \delta\right\}
$$


From the last lemma, $\Psi_{0}(z)$ satisfies these properties:

(i) $a_{1} \leq \Psi_{0}(z) \leq a_{2}$.

(ii) $\triangle \ln \Psi_{0} \geq 0$ for $z \in \mathbb{R}^{2} \backslash \bigcup_{l} D_{l}(a)$.

(iii) $\triangle \ln \Psi_{0} \geq a_{3} \delta^{-2}$ for $z \in A_{l}$.

Note that the $a_{i}$ in the above are positive constants independent of $\lambda$. Let

$$
A=\bigcup_{l} A_{l}
$$

Suppose that $\tau$ is a nonnegative constant. We introduce $\Phi(z)=\Psi_{0}(z) e^{\tau|z|^{2}}$. For $u \in C_{0}^{\infty}\left(\mathbb{R}^{2} \backslash \bigcup_{l} D_{l}(a)\right)$, we assume that $\mathscr{D}$ contains the support of $u$ and $A \subset \mathscr{D} \subset \mathbb{R}^{2} \backslash \cup_{l} D_{l}(a)$. Obviously,

$$
\ln \Phi(z)=\ln \Psi_{0}(z)+\tau|z|^{2} .
$$

Substituting $\Phi$ in Lemma 6 gives that

$$
\int_{\mathscr{D}}|\bar{\partial} u|^{2} \Psi_{0}(z) e^{\tau|z|^{2}} \geq C_{1} \tau \int_{\mathscr{D}}|u|^{2} \Psi_{0}(z) e^{\tau|z|^{2}}+C_{2} \delta^{-2} \int_{A}|u|^{2} e^{\tau|z|^{2}},
$$

where we have used the properties (ii) and (iii) for $\Psi_{0}$. The boundedness of $\Psi_{0}(z)$ yields that

$$
\int_{\mathscr{D}}|\bar{\partial} u|^{2} e^{\tau|z|^{2}} \geq C_{3} \tau \int_{\mathscr{D}}|u|^{2} e^{\tau|z|^{2}}+C_{4} \delta^{-2} \int_{A}|u|^{2} e^{\tau|z|^{2}}
$$

Define the holomorphic function

$$
P(z)=\prod_{l}\left(z-z_{l}\right)
$$

Then $\bar{\partial}(u / P)=\bar{\partial} u / P$. Replacing $u$ by $u / P$ in (3-5), it follows that

$$
\int_{\mathscr{D}}|\bar{\partial} u|^{2}|P|^{-2} e^{\tau|z|^{2}} \geq C_{3} \tau \int_{\mathscr{D}}|u|^{2}|P|^{-2} e^{\tau|z|^{2}}+C_{4} \delta^{-2} \int_{A}|u|^{2}|P|^{-2} e^{\tau|z|^{2}} .
$$

We will establish a Carleman inequality for second-order elliptic equations like (3-2). Write $\tilde{b}(x)=$ $\left(\tilde{b}_{1}(x), \tilde{b}_{2}(x)\right)$. Let

$$
u=\partial f+\frac{1}{2}\left(\tilde{b}_{1}-i \tilde{b}_{2}\right) f
$$

where $f \in C_{0}^{\infty}\left(\mathbb{R}^{2} \backslash \bigcup_{l} D_{l}(a)\right)$ is a real-valued function. Then

$$
\bar{\partial} u=\frac{1}{4}\left[\Delta f+\operatorname{div} \tilde{b} f+\tilde{b} \cdot \nabla f+i\left(\frac{\partial\left(\tilde{b}_{1} f\right)}{\partial y}-\frac{\partial\left(\tilde{b}_{2} f\right)}{\partial x}\right)\right] .
$$


Plugging the above $u$ into (3-6), we obtain

$$
\begin{aligned}
\int_{\mathscr{D}}\left[|\Delta f+\tilde{b} \cdot \nabla f|^{2}\right. & \left.+|\operatorname{div} \tilde{b} f|^{2}+\left|\frac{\partial\left(\tilde{b}_{1} f\right)}{\partial y}-\frac{\partial\left(\tilde{b}_{2} f\right)}{\partial x}\right|^{2}\right]|P|^{-2} e^{\tau|z|^{2}} \\
\geq C_{3} \tau \int_{\mathscr{D}}|\nabla f|^{2}|P|^{-2} e^{\tau|z|^{2}}-C_{3} \tau \int_{\mathscr{D}}|\tilde{b}|^{2}|f|^{2}|P|^{-2} e^{\tau|z|^{2}} & +C_{4} \delta^{-2} \int_{A}|\nabla f|^{2}|P|^{-2} e^{\tau|z|^{2}}-C_{4} \delta^{-2} \int_{A}|\tilde{b}|^{2}|f|^{2}|P|^{-2} e^{\tau|z|^{2}} .
\end{aligned}
$$

If we choose $u=f$ in (3-6), we get

$$
\int_{\mathscr{D}}|\nabla f|^{2}|P|^{-2} e^{\tau|z|^{2}} \geq C_{3} \tau \int_{\mathscr{D}}|f|^{2}|P|^{-2} e^{\tau|z|^{2}} .
$$

Since the norm of $\tilde{b}$ is chosen small enough, it is smaller than $\tau$, which will be chosen large enough. With the aid of (3-8), we can incorporate the terms involving $\tilde{b}$ in the left-hand side of (3-7) into the first term in the right-hand side of (3-7):

$$
\begin{aligned}
\int_{\mathscr{D}} \mid \Delta f & +\left.\tilde{b} \cdot \nabla f\right|^{2}|P|^{-2} e^{\tau|z|^{2}} \\
& \geq C_{5} \tau \int_{\mathscr{D}}|\nabla f|^{2}|P|^{-2} e^{\tau|z|^{2}}+C_{4} \delta^{-2} \int_{A}|\nabla f|^{2}|P|^{-2} e^{\tau|z|^{2}}-C_{4} \delta^{-2} \int_{A}|\tilde{b}|^{2}|f|^{2}|P|^{-2} e^{\tau|z|^{2}} .
\end{aligned}
$$

Furthermore, if $u=f$, the inequality (3-6) implies that

$$
\int_{\mathscr{D}}|\nabla f|^{2}|P|^{-2} e^{\tau|z|^{2}} \geq C_{4} \delta^{-2} \int_{A}|f|^{2}|P|^{-2} e^{\tau|z|^{2}} .
$$

Applying (3-10) to the last term in the right-hand side of (3-9) gives that

$$
\int_{\mathscr{D}}|\triangle f+\tilde{b} \cdot \nabla f|^{2}|P|^{-2} e^{\tau|z|^{2}} \geq C_{6} \tau^{2} \int_{\mathscr{D}}|f|^{2}|P|^{-2} e^{\tau|z|^{2}}+C_{7} \delta^{-2} \int_{A}|\nabla f|^{2}|P|^{-2} e^{\tau|z|^{2}} .
$$

We continue to get a refined estimate for the last term of (3-11). In order to achieve this goal, we need the following hypotheses for the geometry of the disk $D_{l}$ and the parameter $\tau>1$ :

(R1) The radius $\delta$ of each disk $D_{l}$ is less than $a_{4} \tau^{-1}$.

(R2) The distance between any two distinct $z_{l}$ is at least $2 a_{5} \tau^{1 / 2} \delta$.

(R3) The total number of disks $D_{l}$ is at most $a_{6} \tau$.

Under the those assumptions, we have these comparison estimates from [Donnelly and Fefferman 1990a]:

Lemma 8. If $\bar{z}_{1}$ and $\bar{z}_{2}$ are any points in the same component $A_{l}$ of $A$, then:

(i) $a_{7}<e^{\tau\left|\bar{z}_{1}\right|^{2}} / e^{\tau\left|\bar{z}_{2}\right|^{2}}<a_{8}$.

(ii) $a_{9}<\left|P\left(\bar{z}_{1}\right)\right| /\left|P\left(\bar{z}_{2}\right)\right|<a_{10}$. 
We also need the following Poincaré-type inequality on each annulus: if $f \in C^{\infty}\left(A_{l}\right)$ and $f$ vanishes on the inner boundary of $A_{l}$, then

$$
\int_{A_{l}}|\nabla f|^{2} \geq a_{11} \delta^{-2} \int_{A_{l}}|f|^{2}
$$

The proof of (3-12) can be found in [Donnelly and Fefferman 1990a]. Let $z_{l} \in A_{l}$ be chosen arbitrarily. By Lemma 8, it follows that

$$
\int_{A_{l}}|\nabla f|^{2}|P(z)|^{-2} e^{\tau|z|^{2}} \geq C_{8} \sum_{l} e^{\tau\left|z_{l}\right|^{2}}\left|P\left(z_{l}\right)\right|^{-2} \int_{A_{l}}|\nabla f|^{2} .
$$

Since $f \in C_{0}^{\infty}\left(\mathbb{R}^{2} \backslash \bigcup_{l} D_{l}(a)\right)$, the inequality (3-12) yields that

$$
\int_{A_{l}}|\nabla f|^{2}|P(z)|^{-2} e^{\tau|z|^{2}} \geq C_{9} \sum_{l} e^{\tau\left|z_{l}\right|^{2}}\left|P\left(z_{l}\right)\right|^{-2} \delta^{-2} \int_{A_{l}}|f|^{2} .
$$

Using Lemma 8 again, we obtain

$$
\int_{A_{l}}|\nabla f|^{2}|P(z)|^{-2} e^{\tau|z|^{2}} \geq C_{10} \delta^{-2} \int_{A}|f|^{2}|P(z)|^{-2} e^{\tau|z|^{2}} .
$$

Substituting the last inequality into the last term in (3-11) leads to

$$
\int_{\mathscr{D}}|\triangle f+\tilde{b} \cdot \nabla f|^{2}|P|^{-2} e^{\tau|z|^{2}} \geq C_{6} \tau^{2} \int_{\mathscr{D}}|f|^{2}|P|^{-2} e^{\tau|z|^{2}}+C_{11} \delta^{-4} \int_{A}|f|^{2}|P|^{-2} e^{\tau|z|^{2}} .
$$

We summarize the above arguments in the following proposition:

Proposition 9. Assume $f \in C_{0}^{\infty}\left(\mathbb{R}^{2} \backslash \bigcup_{l} D_{l}(a)\right)$. Then:

(i) It holds that

$$
\int_{\mathscr{D}}|\triangle f+\tilde{b} \cdot \nabla f|^{2}|P|^{-2} e^{\tau|z|^{2}} \geq C \tau^{2} \int_{\mathscr{D}}|f|^{2}|P|^{-2} e^{\tau|z|^{2}} .
$$

(ii) If the additional assumptions (R1)-(R3) for $D_{l}$ hold, the stronger inequality (3-13) is satisfied.

\section{Measure of singular sets}

Let $\mathcal{M}$ be a compact smooth surface. In Section 2, we have shown that the Steklov eigenfunction $e_{\lambda}$ vanishes at all points to order at most $C \lambda$. By the implicit function theorem, outside the singular sets, the nodal set is locally a 1-dimensional $C^{1}$ manifold. Adapting the arguments in [Donnelly and Fefferman 1990a] for (3-2), we can estimate those singular points in a quantitative way. We are able to obtain an upper bound for the singular points in terms of the eigenvalue $\lambda$.

Lemma 10. Singular sets consist of at most finitely many points.

Proof. Without loss of generality, we assume that $0 \in \mathscr{Y}_{\lambda}$ and choose normal coordinates $(x, y)$ at the origin. Next we prove there are finitely many singular points in $\bar{M}$. Using Taylor expansion, we expand $v$ locally at the origin. Then $v(x, y)=F_{j}(x, y)+W_{j+1}(x, y)$, where $F_{j}(x, y)$ consists of the leading nonvanishing term with homogenous order $j \geq 2$ and $W_{j+1}(x, y)$ is a higher-order reminder term. Since 
$\Delta v+\bar{b}(z) \cdot \nabla v+\bar{q}(z) v=0$ and the coordinate is normal, we obtain that $\triangle F_{j}=0$. Under polar coordinates, we find that $F_{j}=r^{j}\left(a_{1} \cos (j \theta)+a_{2} \sin (j \theta)\right)$. Obviously, $r^{-1} \partial F_{j} / \partial \theta$ and $\partial F_{j} / \partial r$ have no common zero if $r \neq 0$. Since

$$
\left|\nabla F_{j}\right|^{2}=\left|\frac{\partial F_{j}}{\partial r}\right|^{2}+\frac{1}{r^{2}}\left|\frac{\partial F_{j}}{\partial \theta}\right|^{2}
$$

there exists a small neighborhood $U$ of the origin such that $U \cap \mathscr{S}_{\lambda}=0$. Since $\overline{\mathcal{M}}$ is compact, the lemma follows.

We plan to count the number of singular points in a sufficiently small ball. Let $p \in \bar{M}$. Consider a geodesic ball $\mathbb{B}\left(p, c \lambda^{-1 / 2}\right)$. If $c$ is small enough, then this geodesic ball is contained in a conformal chart. If we choose

$$
w(z)=v\left(c \lambda^{-1 / 2} z\right)
$$

with $c$ sufficiently small then, from (3-1), $w$ satisfies

$$
\triangle w+\hat{b}(x) \cdot \nabla w+\hat{q}(x) w=0 \quad \text { in } \mathbb{B}(0,4),
$$

with $\hat{b}(x)=c \lambda^{-1 / 2} \bar{b}(x)$ and $\hat{q}(x)=c^{2} \lambda^{-1} \bar{q}(x)$. From (2-5), we obtain

$$
\left\{\begin{array}{l}
\|\hat{b}\|_{W^{1, \infty}(\mathbb{B}(0,4))} \leq c \lambda^{1 / 2} \\
\|\hat{q}\|_{W^{1, \infty}(\mathbb{B}(0,4))} \leq c^{2} \lambda
\end{array}\right.
$$

with $c$ sufficiently small.

Next we will count the total order of the vanishing of singular points for $w$ in the sufficiently small ball. We study $w$ in (4-1).

Proposition 11. Suppose $z_{l} \in \mathscr{T}_{\lambda} \cap \mathbb{B}\left(p, c \lambda^{-1 / 2}\right)$, where $v$ vanishes to order $n_{l}+1$. Then $\sum_{l} n_{l} \leq C \lambda$.

Proof. It suffices to count the number of singular points of $w$ in a small Euclidean ball with radius $\frac{1}{10}$ centered at the origin. Suppose that $w$ vanishes to order $n_{l}+1$. Let $n_{l}=m_{l}+1$. We first consider the case $n_{l} \geq 2$. Then $m_{l} \geq 1$. Define the polynomial

$$
P(z)=\prod\left(z-z_{l}\right)^{m_{l}}
$$

with $\left|z_{l}\right|<\frac{1}{10}$. Let $\mathscr{D}=\mathbb{B}(0,2)$ and let $D_{l}$ be small disjoint disks of radius $\delta$ centered at $z_{l}$. If $f \in C_{0}^{\infty}\left(\mathbb{R}^{2} \backslash \bigcup_{l} D_{l}\right)$, the inequality (3-14) in Proposition 9 implies that

$$
\int_{\mathscr{D}}\left(|\triangle f|^{2}+|\hat{b} \cdot \nabla f|^{2}\right)|P|^{-2} e^{d_{1} \lambda|z|^{2}} \geq C_{2} \lambda^{2} \int_{\mathscr{D}}|f|^{2}|P|^{-2} e^{d_{1} \lambda|z|^{2}},
$$

where $\tau=d_{1} \lambda$. We choose a cut-off function $\theta(z)$ such that $\theta w$ has compact support in $\mathscr{D}$. We select the cut-off function $\theta \in C_{0}^{\infty}\left(\mathscr{D} \backslash \bigcup_{l} D_{l}\right)$ with the following properties:

(i) $\theta(z)=1$ if $|z|<\frac{3}{2}$ and $\left|z-z_{l}\right|>2 \delta$.

(ii) $|\nabla \theta|<C_{3}$ and $|\triangle \theta|<C_{4}$ if $|z|>\frac{3}{2}$.

(iii) $|\nabla \theta|<C_{5} \delta^{-1}$ and $|\Delta \theta|<C_{6} \delta^{-2}$ if $\left|z-z_{l}\right|<2 \delta$. 
Substituting $f=\theta w$ into (4-3) yields that

$$
\int_{(|z|<3 / 2) \cup(3 / 2 \leq|z| \leq 2)}|\triangle(\theta w)+\tilde{b} \cdot \nabla(\theta w)|^{2}|P|^{-2} e^{d_{1} \lambda|z|^{2}} \geq C_{2} \lambda^{2} \int_{|z|<3 / 2}|w|^{2}|P|^{-2} e^{d_{1} \lambda|z|^{2}} .
$$

From (4-1),

$$
\triangle(\theta w)+\hat{b} \cdot \nabla(\theta w)=-\hat{q} \theta w+\Delta \theta w+2 \nabla \theta \cdot \nabla w+\hat{b} \cdot \nabla \theta w .
$$

By the assumption on $\theta$, we obtain

$$
|\triangle \theta w|+|\nabla \theta \cdot \nabla w|+|\nabla \theta w| \leq C_{7} \delta^{m_{l}} \quad \text { if }\left|z-z_{l}\right| \leq 2 \delta .
$$

Taking $\delta \rightarrow 0$, by the dominated convergence theorem, we have

$$
\begin{aligned}
c \lambda^{2} \int_{|z|<3 / 2}|w|^{2}|P|^{-2} e^{d_{1} \lambda|z|^{2}}+C_{9}(1+\lambda)^{2} \int_{3 / 2 \leq|z| \leq 2}\left(|w|^{2}+|\nabla w|^{2}\right)|P|^{-2} e^{d_{1} \lambda|z|^{2}} & \\
& \geq C_{10} \lambda^{2} \int_{|z|<3 / 2}|w|^{2}|P|^{-2} e^{d_{1} \lambda|z|^{2}} .
\end{aligned}
$$

Since $c$ is sufficiently small, we can absorb the first term in the left-hand side of (4-4) into the right-hand side. Then

$$
\int_{3 / 2 \leq|z| \leq 2}\left(|w|^{2}+|\nabla w|^{2}\right)|P|^{-2} e^{d_{1} \lambda|z|^{2}} \geq C_{11} \int_{|z| \leq 1 / 2}|w|^{2}|P|^{-2} e^{d_{1} \lambda|z|^{2}} .
$$

Obviously, it follows that

$$
\max _{|z| \geq 3 / 2}|P|^{-2} \int_{3 / 2 \leq|z| \leq 2}\left(|w|^{2}+|\nabla w|^{2}\right) e^{d_{1} \lambda|z|^{2}} \geq C_{11}\left(\min _{|z| \leq 1 / 2}|P|^{-2}\right) \int_{|z| \leq 1 / 2}|w|^{2} .
$$

By standard elliptic theory, the last inequality implies

$$
\max _{|z| \geq 3 / 2}|P|^{-2} e^{d_{2} \lambda} \int_{|z| \leq 5 / 2}|w|^{2} \geq C_{11}\left(\min _{|z| \leq 1 / 2}|P|^{-2}\right) \int_{|z| \leq 1 / 2}|w|^{2}
$$

We claim that

$$
e^{d_{3} \sum m_{l}} \leq \frac{\min _{|z| \leq 1 / 2}|P|^{-2}}{\max _{|z| \geq 3 / 2}|P|^{-2}} .
$$

To prove (4-8), it suffices to verify

$$
e^{-d_{4} \sum m_{l}} \min _{|z| \geq 3 / 2}|P| \geq \max _{|z| \leq 1 / 2}|P|
$$

away from the singular point $z_{l}$. Clearly,

$$
\max _{|z| \leq 1 / 2}|P| \leq\left(\frac{1}{2}\right)^{\sum m_{l}} .
$$

Since $z_{l} \in \mathbb{B}\left(0, \frac{1}{10}\right)$, we have

$$
\left(\frac{3}{4}\right)^{\sum m_{l}} \leq \min _{|z| \geq 3 / 2}|P| .
$$


Combining the last two inequalities, we obtain (4-9). The claim is shown. Let's return to (4-7); we get

$$
\frac{\min _{|z| \leq 1 / 2}|P|^{-2}}{\max _{|z| \geq 3 / 2}|P|^{-2}} \leq \frac{e^{d_{5} \lambda} \int_{|z| \leq 5 / 2}|w|^{2}}{C_{11} \int_{|z| \leq 1 / 2}|w|^{2}} \leq e^{d_{6} \lambda}
$$

where we applied doubling estimates in the last inequality. Thanks to (4-8), we obtain

$$
\sum m_{l} \leq d_{7} \lambda
$$

Since $n_{l}=m_{l}+1 \leq 2 m_{l}$, we complete the lemma for $n_{l} \geq 2$.

If the vanishing order for the singular point is two, i.e., $n_{l}=1$. We consider $Q(z)=\prod\left(z-z_{l}\right)^{n_{l} / 2}$ instead of $P(z)$. In this case, $Q(z)$ may not be defined as a single-valued holomorphic function on $\mathscr{b}$. We pass to a finite-branched cover of the disk $\mathscr{D}$ punctured at $z_{l}$. The Carleman estimates in the previous sections still work. The same conclusion will follow.

Based on the vanishing order estimate in Proposition 11, we are able to count the number of singular points.

Proof of Theorem 2. We cover the double manifold $\bar{M}$ by geodesic balls with radius $C \lambda^{-1 / 2}$. Since $\bar{M}$ is compact, the order of those balls is $C \lambda$. From Proposition 11, the conclusion in Theorem 2 follows.

Remark 12. Thanks to Proposition 11 , we can actually show a stronger result. Let $z_{l} \in \mathcal{M}$ be a singular point with vanishing order $n_{l}+1$. Then $\sum_{l} n_{l} \leq C \lambda^{2}$.

\section{Growth of eigenfunctions}

In this section, we will show that the eigenfunctions do not grow rapidly on too many small balls. We still restrict $v$ to the small geodesic ball $\mathbb{B}\left(p, c \lambda^{-1 / 2}\right)$ in the conformal chart. Let $w(z)=v\left(c \lambda^{-1 / 2} z\right)$. Then $w$ satisfies the elliptic equation (4-1) with assumptions (4-2) in a Euclidean ball of radius 4 centered at the origin. If we suppose that $w$ grows rapidly, that is,

$$
C_{1} \int_{(1-3 a) \delta \leq\left|z-z_{l}\right| \leq(1-3 a / 4) \delta} w^{2} \leq \int_{(1-3 a / 2) \delta \leq\left|z-z_{l}\right| \leq(1-a) \delta} w^{2}
$$

for all $l$ and some large $C_{1}$, then the following proposition is valid:

Proposition 13. Suppose $D_{l}$ are disks contained in a Euclidean ball of radius $\frac{1}{30}$ centered at the origin. Furthermore, assume that

(R1) $\delta<d_{1} \lambda^{-1}$, and

(R2) $\left|z_{l}-z_{k}\right|>d_{2} \lambda^{1 / 2} \delta$ when $l \neq k$.

If (5-1) holds for all $l$, the number of disks $D_{l}$ is less than $d_{3} \lambda$.

Proof. We will use the stronger Carleman estimates in (3-13) in Proposition 9. We prove it by contradiction. Suppose that the collection $D_{l}=\left\{z|| z-z_{l} \mid \leq \delta\right\}$ are disjoint disks satisfying the hypotheses (R1)-(R3) in Section 3. Without loss of generality, we require that all the $D_{l}$ are in a ball centered at the origin with radius $\frac{1}{30}$. As before, $D_{l}(a)=\left\{z|| z-z_{l} \mid \leq(1-2 a) \delta\right\}$, where $a$ is a suitably small positive constant. Let 
$\mathscr{D}$ be a ball centered at the origin with radius 2 . We choose a cut-off function $\theta \in C_{0}^{\infty}\left(\mathscr{D} \backslash \bigcup_{l} D_{l}\right)$ and assume $\theta(z)$ satisfies the following properties:

(i) $\theta(z)=1$ if $|z|<1$ and $\left|z-z_{l}\right|>\left(1-\frac{3}{2} a\right) \delta$ for all $l$.

(ii) $|\nabla \theta|+|\triangle \theta|<C_{2}$ if $|z|>1$.

(iii) $|\nabla \theta|<C_{3} \delta^{-1}$ and $|\triangle \theta|<C_{4} \delta^{-2}$ if $\left|z-z_{l}\right|<\left(1-\frac{3}{2} a\right) \delta$.

Substituting $f=\theta w$ into (3-13) gives that

$$
\int_{\mathscr{D}}|\triangle(\theta w)+\hat{b} \cdot \nabla(\theta w)|^{2}|P|^{-2} e^{d_{4} \lambda|z|^{2}} \geq C_{5} \lambda^{2} \int_{\mathscr{D}}|\theta w|^{2}|P|^{-2} e^{d_{4} \lambda|z|^{2}}+C_{6} \delta^{-4} \int_{A}|\theta w|^{2}|P|^{-2} e^{d_{4} \lambda|z|^{2}} .
$$

We also assume $\tau=d_{4} \lambda$. Recall that $A=\bigcup_{l} A_{l}$ and $A_{l}=\left\{z|(1-2 a) \delta \leq| z-z_{l} \mid \leq(1-a) \delta\right\}$. We first consider the integral in the left-hand side of the last inequality. Again, by (4-1),

$$
\triangle(\theta w)+\hat{b} \cdot \nabla(\theta w)=-\hat{q} \theta w+\triangle \theta w+2 \nabla \theta \cdot \nabla w+\hat{b} \cdot \nabla \theta w .
$$

Thus,

$$
|\triangle(\theta w)+\hat{b} \cdot \nabla(\theta w)|^{2} \leq C\left(c \lambda^{2} \theta^{2} w^{2}+|\triangle \theta|^{2} w^{2}+|\nabla \theta|^{2}|\nabla w|^{2}+c \lambda|\nabla \theta|^{2} w^{2}\right)
$$

where $c$ is sufficiently small. We will absorb the term involving $\theta^{2} w^{2}$ into the right-hand side of (5-2). Since $c$ is small enough, we get

$$
\begin{aligned}
\int_{\mathscr{D}}\left(|\triangle \theta|^{2} w^{2}+c|\nabla \theta|^{2} w^{2}+|\nabla \theta|^{2}|\nabla w|^{2}\right)|P|^{-2} e^{d_{4} \lambda|z|^{2}} & \\
& \geq C_{7} \lambda^{2} \int_{\mathscr{D}}|\theta w|^{2}|P|^{-2} e^{d_{4} \lambda|z|^{2}}+C_{8} \delta^{-4} \int_{A}|\theta w|^{2}|P|^{-2} e^{d_{4} \lambda|z|^{2}} .
\end{aligned}
$$

Using the properties of $\theta(z)$ and taking into account that each $D_{l}$ lies in the ball centered at the origin with radius $\frac{1}{30}$, we obtain

$$
\begin{aligned}
\int_{\mathscr{D}}(\mid & \left.\left.\Delta \theta\right|^{2} w^{2}+c|\nabla \theta|^{2} w^{2}+|\nabla \theta|^{2}|\nabla w|^{2}\right)|P|^{-2} e^{d_{4} \lambda|z|^{2}} \\
& \geq C_{7} \lambda^{2} \int_{1 / 4 \leq|z| \leq 1 / 2}|w|^{2}|P|^{-2} e^{d_{4} \lambda|z|^{2}}+C_{9} \delta^{-4} \sum_{l} \int_{(1-3 a / 2) \delta \leq\left|z-z_{l}\right| \leq(1-a) \delta}|w|^{2}|P|^{-2} e^{d_{4} \lambda|z|^{2}} .
\end{aligned}
$$

Next we want to control the left-hand side of the last inequality. Write

$$
\int_{\mathscr{D}}\left(|\triangle \theta|^{2} w^{2}+c|\nabla \theta|^{2} w^{2}+|\nabla \theta|^{2}|\nabla w|^{2}\right)|P|^{-2} e^{d_{4} \lambda|z|^{2}}=I+\sum_{l} I_{l},
$$

where

$$
\begin{aligned}
I & =\int_{1 \leq|z| \leq 2}\left(|\Delta \theta|^{2} w^{2}+c|\nabla \theta|^{2} w^{2}+|\nabla \theta|^{2}|\nabla w|^{2}\right)|P|^{-2} e^{d_{4} \lambda|z|^{2}}, \\
I_{l} & =\int_{(1-2 a) \delta \leq\left|z-z_{l}\right| \leq(1-3 a / 2) \delta}\left(|\triangle \theta|^{2} w^{2}+c|\nabla \theta|^{2} w^{2}+|\nabla \theta|^{2}|\nabla w|^{2}\right)|P|^{-2} e^{d_{4} \lambda|z|^{2}} .
\end{aligned}
$$


By standard elliptic estimates,

$$
I \leq e^{d_{5} \lambda} \max _{|z| \geq 1}|P|^{-2} \int_{3 / 4 \leq|z| \leq 5 / 2} w^{2} .
$$

Similarly, via elliptic estimates,

$$
I_{l} \leq C_{10} \delta^{-4}\left(\max _{A_{l}}|P|^{-2} e^{d_{4} \lambda|z|}\right) \int_{(1-3 a) \delta \leq\left|z-z_{l}\right| \leq(1-3 a / 4) \delta} w^{2} .
$$

Thanks to Lemma 8,

$$
I_{l} \leq C_{11} \delta^{-4}\left(\min _{A_{l}}|P|^{-2} e^{d_{4} \lambda|z|}\right) \int_{(1-3 a) \delta \leq\left|z-z_{l}\right| \leq(1-3 a / 4) \delta} w^{2} .
$$

Combining these inequalities together in (5-4) leads to

$$
\begin{aligned}
& e^{d_{5} \lambda} \max _{|z| \geq 1}|P|^{-2} \int_{3 / 4 \leq|z| \leq 5 / 2} w^{2}+C_{11} \delta^{-4} \sum_{l}\left(\min _{A_{l}}|P|^{-2} e^{d_{4} \lambda|z|}\right) \int_{(1-3 a) \delta \leq\left|z-z_{l}\right| \leq(1-3 a / 4) \delta} w^{2} \\
& \geq C_{12} \min _{|z| \leq 1 / 2}|P|^{-2} \int_{1 / 4 \leq|z| \leq 1 / 2}|w|^{2}+C_{13} \delta^{-4} \sum_{l} \min _{A_{l}}\left(|P|^{-2} e^{d_{4} \lambda|z|^{2}}\right) \int_{(1-3 a / 2) \delta \leq\left|z-z_{l}\right| \leq(1-a) \delta} w^{2} .
\end{aligned}
$$

Performing similar arguments as for (4-8) shows that

$$
\min _{|z| \leq 1 / 2}|P|^{-2}>\max _{|z| \geq 1}|P|^{-2} e^{d_{5} \sum_{l} m_{l}} .
$$

If the number of the $D_{l}$ is $d_{3} \lambda$, then

$$
\min _{|z| \leq 1 / 2}|P|^{-2}>\max _{|z| \geq 1}|P|^{-2} e^{d_{6} \lambda}
$$

We claim that

$$
e^{C_{14} \lambda} \int_{1 / 4 \leq|z| \leq 1 / 2} w^{2} \geq \int_{3 / 4 \leq|z| \leq 5 / 2} w^{2} .
$$

We prove the claim by doubling estimates shown in Proposition 5 . We choose a ball $\mathbb{B}\left(x_{0}, \frac{1}{8}\right) \subset$ $\left\{z\left|\frac{1}{4} \leq\right| z \mid \leq \frac{1}{2}\right\}$. It is clear that

$$
\int_{1 / 4 \leq|z| \leq 1 / 2} w^{2} \geq \int_{\mathbb{B}\left(x_{0}, 1 / 8\right)} w^{2} .
$$

Using doubling estimates, we have

$$
e^{C_{15} \lambda} \int_{\mathbb{B}\left(x_{0}, 1 / 8\right)} w^{2} \geq \int_{\mathbb{B}\left(x_{0}, 2 / 8\right)} w^{2} .
$$

By finite iterations, we can find a large ball $\mathbb{B}\left(x_{0}, 3\right)$ that contains $\left\{z\left|\frac{3}{4} \leq\right| z \mid \leq \frac{5}{2}\right\}$. This yields that

$$
\int_{\mathbb{B}\left(x_{0}, 3\right)} w^{2} \geq \int_{3 / 4 \leq|z| \leq 5 / 2} w^{2} .
$$

Then the combination of these inequalities verifies the claim. 
If we choose $d_{3}$ suitably large, since the number of disks $D_{l}$ is $d_{3} \lambda$, also $d_{6}$ is suitably large. From the inequalities (5-10) and (5-11), it follows that

$$
e^{d_{5} \lambda} \max _{|z| \geq 1}|P|^{-2} \int_{3 / 4 \leq|z| \leq 5 / 2} w^{2}<C_{12} \min _{|z| \leq 1 / 2}|P|^{-2} \int_{1 / 4 \leq|z| \leq 1 / 2} w^{2} .
$$

This contradicts the estimates (5-1) and (5-9). The proposition is proved.

\section{Growth estimates and nodal length}

The purpose of this section is to find the connection between growth of eigenfunctions and nodal length. A suitable small growth in $L^{2}$ norm implies an upper bound on nodal length. We consider the second-order elliptic equations

$$
\triangle \bar{w}+b^{*} \cdot \nabla \bar{w}+q^{*} \bar{w}=0 \quad \text { in } \mathbb{B}(0,4) .
$$

Assume that there exists a positive constant $C$ such that $\left\|b^{*}\right\|_{W^{1, \infty}} \leq C$ and $\left\|q^{*}\right\|_{W^{1, \infty}} \leq C$. The following lemma relies on the Carleman estimates in Lemma 4. Suppose $\epsilon_{1}$ is a sufficiently small positive constant.

Lemma 14. Suppose that $w$ satisfies the growth estimate

$$
\int_{(1-3 a / 2) \epsilon_{0}<r<(1-a) \epsilon_{0}} \bar{w}^{2} \leq C_{3} \int_{(1-3 a) \epsilon_{0}<r<(1-4 a / 3) \epsilon_{0}} \bar{w}^{2},
$$

where $a$ and $\epsilon_{0}$ are fixed small constants. Then, for $0<\epsilon_{1}<\frac{1}{100} \epsilon_{0}$, we have

$$
\max _{r \leq \epsilon_{1}}|\bar{w}| \geq C_{4}\left(\frac{\epsilon_{1}}{\epsilon_{0}}\right)^{C_{5}}\left(f_{\mathbb{B}\left(0,(1-4 / 3 a) \epsilon_{0}\right)} \bar{w}^{2}\right)^{\frac{1}{2}},
$$

where $f$ denotes the average of the integration.

Proof. We select a radial cut-off function $\theta \in C_{0}^{\infty}\left(\frac{1}{2} \epsilon_{1}<r<\left(1-\frac{11}{10} a\right) \epsilon_{0}\right)$ that satisfies the properties:

(i) $\theta(r)=1$ for $\frac{3}{4} \epsilon_{1}<r<\left(1-\frac{10}{9} a\right) \epsilon_{0}$.

(ii) $|\nabla \theta|+|\triangle \theta| \leq C_{6}$ for $r>\left(1-\frac{10}{9} a\right) \epsilon_{0}$.

(iii) $|\nabla \theta| \leq C_{7} \epsilon_{1}^{-1}$ and $|\triangle \theta|<C_{8} \epsilon_{1}^{-2}$ for $r \leq \frac{3}{4} \epsilon_{1}$.

From (6-1), we get

$$
\triangle(\theta \bar{w})+b^{*} \cdot \nabla(\theta \bar{w})+q^{*} \theta \bar{w}=\Delta \theta \bar{w}+2 \nabla \theta \cdot \nabla \bar{w}+b^{*} \cdot \nabla \theta \bar{w} .
$$

Assume that $\tau>C$ is large enough. Substituting $u=\theta \bar{w}$ in Lemma 4 yields that

$$
C_{2} \tau^{3} \int r^{\epsilon} e^{2 \tau \phi(r)} \theta^{2} \bar{w}^{2} d r d \omega \leq I
$$

where

$$
I=\int r^{4} e^{2 \tau \phi(r)}\left|\triangle \theta \bar{w}+2 \nabla \theta \cdot \nabla \bar{w}+b^{*} \cdot \nabla \theta \bar{w}\right|^{2} d r d \omega
$$


Note that $\phi(r)$ is a decreasing function. Furthermore, by the assumptions on $\theta(z)$, we obtain

$$
\begin{aligned}
I \leq e^{2 \tau \phi\left(\epsilon_{1} / 2\right)} \int_{\epsilon_{1} / 2<r<3 \epsilon_{1} / 4}\left|\Delta \theta \bar{w}+2 \nabla \theta \cdot \nabla \bar{w}+b^{*} \cdot \nabla \theta \bar{w}\right|^{2} r d r d \omega \\
\quad+e^{2 \tau \phi\left((1-10 a / 9) \epsilon_{0}\right)} \int_{(1-10 a / 9) \epsilon_{0}<r<(1-11 a / 10) \epsilon_{0}}\left|\Delta \theta \bar{w}+2 \nabla \theta \cdot \nabla \bar{w}+b^{*} \cdot \nabla \theta \bar{w}\right|^{2} r d r d \omega .
\end{aligned}
$$

By standard elliptic estimates, we derive that

$$
I \leq C_{9} e^{2 \tau \phi\left(\epsilon_{1} / 2\right)} \int_{\epsilon_{1} / 4<r<\epsilon_{1}} \bar{w}^{2} r d r d \omega+C_{10} e^{2 \tau \phi\left((1-10 a / 9) \epsilon_{0}\right)} \int_{(1-3 a / 2) \epsilon_{0}<r<(1-a) \epsilon_{0}} \bar{w}^{2} r d r d \omega .
$$

Taking the inequality (6-4) and assumptions of $\theta$ into account, we have

$$
\begin{aligned}
C_{10} e^{2 \tau \phi\left((1-10 a / 9) \epsilon_{0}\right)} \int_{(1-3 a / 2) \epsilon_{0}<r<(1-a) \epsilon_{0}} \bar{w}^{2} r d r d \omega+C_{9} e^{2 \tau \phi\left(\epsilon_{1} / 2\right)} \int_{\epsilon_{1} / 4<r<\epsilon_{1}} \bar{w}^{2} r d r d \omega \\
\geq C_{2} \tau^{3} \int_{3 \epsilon_{1} / 4<r<(1-10 a / 9) \epsilon_{0}} r^{\epsilon} e^{2 \tau \phi(r)} \bar{w}^{2} d r d \omega \\
\geq C_{2} \tau^{3}\left(\left(1-\frac{10}{9} a\right) \epsilon_{0}\right) \int_{3 \epsilon_{1} / 4<r<(1-10 a / 9) \epsilon_{0}} e^{2 \tau \phi(r)} \bar{w}^{2} r d r d \omega .
\end{aligned}
$$

Since $\epsilon$ and $\epsilon_{0}$ are fixed positive constants, taking $\tau$ large enough we obtain

$$
\frac{1}{2} C_{2} \tau^{3}\left(\left(1-\frac{10}{9} a\right) \epsilon_{0}\right)^{\epsilon-1}>C_{10} .
$$

Taking the hypothesis (6-2) into consideration, we can incorporate the first term in the left-hand side of (6-6) into the right-hand side. It follows that

$$
C_{9} e^{2 \tau \phi\left(\epsilon_{1} / 2\right)} \int_{\epsilon_{1} / 4<r<\epsilon_{1}} \bar{w}^{2} r d r d \omega \geq C_{10} e^{2 \tau \phi\left((1-10 a / 9) \epsilon_{0}\right)} \int_{3 \epsilon_{1} / 4<r<(1-10 a / 9) \epsilon_{0}} \bar{w}^{2} r d r d \omega .
$$

Fix such a $\tau$; adding the term

$$
e^{2 \tau \phi\left((1-10 a / 9) \epsilon_{0}\right)} \int_{r<3 \epsilon_{1} / 4} \bar{w}^{2} r d r d \omega
$$

to both sides of the last inequality yields that

$$
e^{2 \tau \phi\left(\epsilon_{1} / 2\right)} \int_{r<\epsilon_{1}} \bar{w}^{2} r d r d \omega \geq C_{11} e^{2 \tau \phi\left((1-10 a / 9) \epsilon_{0}\right)} \int_{r<(1-4 a / 3) \epsilon_{0}} \bar{w}^{2} r d r d \omega,
$$

where we have used the fact that $\phi$ is decreasing. Straightforward calculations show that

$$
e^{2 \tau\left(\phi\left((1-10 a / 9) \epsilon_{0}\right)-\phi\left(\epsilon_{1} / 2\right)\right)} \geq C_{13}\left(\frac{\epsilon_{1}}{\epsilon_{0}}\right)^{C_{12}}
$$

Thus,

$$
\int_{r<\epsilon_{1}} \bar{w}^{2} r d r d \omega \geq C_{13}\left(\frac{\epsilon_{1}}{\epsilon_{0}}\right)^{C_{12}} \int_{r<(1-4 a / 3) \epsilon_{0}} \bar{w}^{2} r d r d \omega .
$$

This completes the lemma. 
Our next goal is to find the relation between Lemma 14 and nodal length. We assume that the estimate (6-2) exists. Then the conclusion (6-3) in Lemma 14 holds. For $\epsilon_{1} \leq \frac{1}{100} \epsilon$, if $|z|<\epsilon_{1}$ then using Taylor's expansion gives that

$$
\left|\bar{w}(z)-\sum_{|\alpha| \leq C_{5}} \frac{1}{\alpha !} \frac{\partial^{\alpha} \bar{w}}{\partial z^{\alpha}}(0) z^{\alpha}\right| \leq \sup _{|z| \leq \epsilon_{1}|\alpha|=C_{5}+1} \sup _{1}\left|\frac{\partial^{\alpha} \bar{w}}{\partial z^{\alpha}}(z)\right| \epsilon_{1} C_{5}+1,
$$

where $\alpha=\left(\alpha_{1}, \alpha_{2}\right)$ and $\partial / \partial z^{\alpha}=\partial / \partial z_{1}{ }^{\alpha_{1}} \cdot \partial / \partial z_{2}{ }^{\alpha_{2}}$. To control the right-hand side of the last inequality, by elliptic estimates and a rescaling argument we have

$$
\left|\bar{w}(z)-\sum_{|\alpha| \leq C_{5}} \frac{1}{\alpha !} \frac{\partial^{\alpha} \bar{w}}{\partial z^{\alpha}}(0) z^{\alpha}\right| \leq d_{2}\left(f_{\mathbb{B}\left(0,(1-4 a / 3) \epsilon_{0}\right)} \bar{w}^{2}\right)^{\frac{1}{2}}\left(\frac{\epsilon_{1}}{\epsilon_{0}}\right)^{C_{5}+1} .
$$

Using the estimate (6-3) in Lemma 14, we get

$$
\left|\bar{w}(z)-\sum_{|\alpha| \leq C_{5}} \frac{1}{\alpha !} \frac{\partial^{\alpha} \bar{w}}{\partial z^{\alpha}}(0) z^{\alpha}\right| \leq d_{3}\left(\frac{\epsilon_{1}}{\epsilon_{0}}\right) \max _{|z| \leq \epsilon_{1}}|\bar{w}| .
$$

Choosing $\epsilon_{1} / \epsilon_{0}$ sufficiently small, by the triangle inequality we obtain

$$
\sup _{|\alpha| \leq C_{5}}\left|\frac{\partial^{\alpha} \bar{w}}{\partial z^{\alpha}}(0)\right| \epsilon_{1}^{|\alpha|} \geq d_{4} \max _{|z| \leq \epsilon_{1}}|\bar{w}| .
$$

Applying again the estimate (6-3) to the right-hand side of the last inequality yields that

$$
\sup _{|\alpha| \leq C_{5}}\left|\frac{\partial^{\alpha} \bar{w}}{\partial z^{\alpha}}(0)\right| \epsilon_{0}^{|\alpha|} \geq d_{5}\left(f_{\mathbb{B}\left(0,(1-4 a / 3) \epsilon_{0}\right)} \bar{w}^{2}\right)^{\frac{1}{2}} .
$$

By standard elliptic estimates, we also have

$$
\sup _{|z| \leq \epsilon_{0} / 2|\alpha| \leq C_{5}+1} \sup _{\left|\frac{\partial^{\alpha} \bar{w}}{\partial z^{\alpha}}(z)\right| \epsilon_{0}|\alpha|} \leq d_{6}\left(f_{\mathbb{B}\left(0,(1-4 a / 3) \epsilon_{0}\right)} \bar{w}^{2}\right)^{\frac{1}{2}} .
$$

The basic relationship between derivatives and nodal length in two dimensions is shown in [Donnelly and Fefferman 1990a].

Lemma 15. Suppose that $\bar{w}$ satisfies (6-10) and (6-11). Then

$$
H^{1}\left(z|| z \mid \leq d_{7} \bar{\epsilon} \text { and } \bar{w}(z)=0\right) \leq d_{8} \bar{\epsilon} .
$$

With the aid of the last lemma, we can readily obtain an upper nodal length estimate.

Proposition 16. Let $\bar{w}$ be the solution of (6-1). Suppose that $\bar{\epsilon} \leq \epsilon_{0}$ and $w$ satisfies the growth condition

$$
\int_{(1-3 a / 2) \bar{\epsilon}<r<(1-a) \bar{\epsilon}} \bar{w}^{2} \leq C_{3} \int_{(1-3 a) \bar{\epsilon}<r<(1-4 a / 3) \bar{\epsilon}} \bar{w}^{2} .
$$

Then

$$
H^{1}\left(z|| z \mid \leq d_{9} \bar{\epsilon} \text { and } \bar{w}(z)=0\right) \leq d_{10} \bar{\epsilon}
$$


Proof. Since the inequalities (6-10) and (6-11) can be derived from (6-12) by Lemma 14, the proposition follows from the last lemma.

\section{Total nodal length}

As Proposition 13 indicates, the eigenfunctions cannot grow rapidly on too many small balls. If they grow slowly, we have an upper bound on the local length of nodal sets by Proposition 16. In this section, we will link these two arguments together. To achieve it, we will employ a process of repeated subdivision and selection of squares. The idea is inspired by [Donnelly and Fefferman 1990a].

Assume that $\mathbb{B}\left(p, c \lambda^{-1 / 2}\right)$ is a geodesic ball of the double manifold $\bar{M}$. Choosing $c$ to be small, it is contained in a conformal chart. Let $w(z)=v\left(c \lambda^{-1 / 2} z\right)$ with $c$ sufficiently small. We know that $w$ satisfies

$$
\triangle w+\hat{b}(x) \cdot \nabla w+\hat{q}(x) w=0 \quad \text { in } \mathbb{B}(0,4) .
$$

We consider the square $P=\left\{(x, y) \mid \max (|x|,|y|) \leq \frac{1}{60}\right\}$ in $\mathbb{B}(0,4)$ and divide it into a grid of closed squares $P_{l}$ with side $\delta \leq a_{1} \lambda^{-1}$. If (5-1) holds for some point $z_{l} \in P_{l}$ and for some sufficiently large $C_{1}$, we call $P_{l}$ a square of rapid growth. With the aid of Proposition 13, we are able to obtain the following result:

Lemma 17. There are at most $C \lambda^{2}$ squares with side $\delta$ where $w$ is of rapid growth.

Proof. Let $I_{1}$ be the collection of those indices $l$ for which $P_{l}$ is a square of rapid growth. For each $l \in I_{1}$, there exists some point $z_{l} \in P_{l}$ such that (5-1) holds. Let $\left|I_{1}\right|$ denote the cardinality of $I_{1}$. Define

$$
P_{l}^{*}=\left\{z|| z-z_{l} \mid<d_{1} \delta \lambda^{1 / 2}\right\} .
$$

The collection of disks $P_{l}^{*}$ covers the collection of squares $P_{l}$ for $l \in I_{1}$. We choose a maximal collection of disjoint disks of $P_{l}^{*}$ and denote it as $I_{2}$. If $l \in I_{2}$, we define

$$
P_{l}^{* *}=\left\{z|| z-z_{l} \mid<4 d_{1} \delta \lambda^{1 / 2}\right\} .
$$

Since the collection of disks in $I_{2}$ is maximal and they are disjoint, we obtain that

$$
\bigcup_{l \in I_{2}} P_{l}^{* *} \supseteq \bigcup_{l \in I_{1}} P_{l}^{*} \supseteq \bigcup_{l \in I_{1}} P_{l} .
$$

Thus,

$$
\left|I_{2}\right| \times 16 d_{1}^{2} \delta^{2} \lambda \geq\left|I_{1}\right| \delta^{2}
$$

which implies

$$
\left|I_{2}\right| \lambda \geq d_{2}\left|I_{1}\right|
$$

Recall from Proposition 13 that $\left|I_{2}\right| \leq d_{3} \lambda$. Therefore, we obtain the desirable estimate $\left|I_{1}\right| \leq d_{4} \lambda^{2}$.

Now we introduce an iterative process of bisecting squares. We begin by dividing the square into a grid of squares $P_{l}(1)$ with side $\delta(1)=a_{1} \lambda^{-1}$, then separate them into two categories $R_{l}(1)$ and $S_{l}(1)$. $R_{l}(1)$ are those where $w$ is of rapid growth and $S_{l}(1)$ are those where (5-1) fails for $w$. We continue to bisect each square $R_{l}(1)$ to obtain squares $P_{l}(2)$ with side $\delta(2)=\frac{1}{2} \delta(1)$. Again, we split $P_{i}(2)$ into the 
subcollection $R_{l}(2)$ with rapid growth and $S_{l}(2)$ with slow growth. We repeat the process at each step $k$. Then there are squares $R_{l}(k)$ and $S_{l}(k)$ with $\delta(k)=\frac{1}{2^{k}} \delta(1)$. We count the number of $R_{l}(k)$ and $S_{l}(k)$ at step $k$ :

Lemma 18. (i) The number of squares $R_{l}(k)$ is at most $C_{2} \lambda^{2}$.

(ii) The number of squares $S_{l}(k)$ is at most $C_{3} \lambda^{2}$.

Proof. The conclusion (i) follows directly from Lemma 17. We only need to show (ii). If $k=1$, the conclusion (ii) follows because the total number of squares is at most of order $\lambda^{2}$. If $k \geq 2$ then, by construction of those squares,

$$
\left|S_{l}(k)\right| \leq 4\left|R_{l}(k-1)\right| \leq C_{4} \lambda^{2},
$$

where we have used (i) in the last inequality. The lemma is done.

The next lemma tells that almost every point lies in some $R_{l}(k)$ with slow growth. It is Lemma 6.3 in [Donnelly and Fefferman 1990a].

Lemma 19. $\bigcup_{k, l} S_{l}(k)$ covers the square $P$ except for singular points $\mathscr{S}=\{z \in P \mid w(x)=0, \nabla w=0\}$.

We are ready to give the proof of Theorem 3.

Proof of Theorem 3. Consider $\bar{w}(z)=w\left(z_{l}+\epsilon_{0}^{-1} \delta(k) z\right)$. Then $\bar{w}(z)$ satisfies (6-1). Choosing a finite collection of $z_{l} \in S_{l}(k)$ and applying Proposition 16, we have

$$
H^{1}\left(z \mid w(z)=0 \text { and } z \in S_{l}(k)\right) \leq C_{5} 2^{-k} \lambda^{-1} .
$$

Furthermore, thanks to Lemma 19,

$$
\begin{aligned}
H^{1}\left(z \mid w(z)=0 \text { and } \max (|x|,|y|) \leq \frac{1}{60}\right) & \leq \sum_{l, k} H^{1}\left(z \mid w(z)=0 \text { and } z \in S_{l}(k)\right) \\
& \leq \lambda^{2} \sum_{k} C_{5} 2^{-k} \lambda^{-1} \leq C_{6} \lambda
\end{aligned}
$$

where we have used (ii) in Lemma 18 and (7-2). Since $w(z)=v\left(c \lambda^{-1 / 2} z\right)$, by the rescaling argument, we obtain

$$
H^{1}\left(\{v(z)=0\} \cap \mathbb{B}\left(p, c \lambda^{-1 / 2}\right)\right) \leq C_{6} \lambda^{1 / 2} .
$$

Finally, covering $\bar{M}$ with order $\lambda$ of geodesic balls with radius $c \lambda^{-1 / 2}$, we readily deduce that

$$
H^{1}(z \in \bar{M} \mid v(z)=0) \leq C_{7} \lambda^{3 / 2} .
$$

Thus, so is $H^{1}\left(\mathcal{N}_{\lambda}\right)$.

\section{Acknowledgements}

It is my pleasure to thank Professor Christopher D. Sogge for helpful discussions about this topic and guidance in the area of eigenfunctions. I would like to thank X. Wang for many fruitful conversations. I would also like to express sincere thanks to the anonymous referee for insightful and constructive comments. 


\section{References}

[Bakri and Casteras 2014] L. Bakri and J.-B. Casteras, "Quantitative uniqueness for Schrödinger operator with regular potentials", Math. Methods Appl. Sci. 37:13 (2014), 1992-2008. MR 3245115 Zbl 1301.35190

[Bellová and Lin 2015] K. Bellová and F.-H. Lin, "Nodal sets of Steklov eigenfunctions", Calc. Var. Partial Differential Equations 54:2 (2015), 2239-2268. MR 3396451 Zbl 1327.35263

[Brüning 1978] J. Brüning, “Über Knoten von Eigenfunktionen des Laplace-Beltrami-Operators”, Math. Z. 158:1 (1978), 15-21. MR 0478247 Zbl 0349.58012

[Calderón 1980] A.-P. Calderón, "On an inverse boundary value problem”, pp. 65-73 in Seminar on numerical analysis and its applications to continuum physics (Rio de Janeiro, 1980), Soc. Brasil. Mat., Rio de Janeiro, 1980. MR 590275

[Colding and Minicozzi 2011] T. H. Colding and W. P. Minicozzi, II, "Lower bounds for nodal sets of eigenfunctions", Comm. Math. Phys. 306:3 (2011), 777-784. MR 2825508 Zbl 1238.58020

[Dong 1992] R.-T. Dong, "Nodal sets of eigenfunctions on Riemann surfaces", J. Differential Geom. 36:2 (1992), $493-506$. MR 1180391 Zbl 0776.53024

[Donnelly and Fefferman 1988] H. Donnelly and C. Fefferman, "Nodal sets of eigenfunctions on Riemannian manifolds", Invent. Math. 93:1 (1988), 161-183. MR 943927 Zbl 0659.58047

[Donnelly and Fefferman 1990a] H. Donnelly and C. Fefferman, "Nodal sets for eigenfunctions of the Laplacian on surfaces", $J$. Amer. Math. Soc. 3:2 (1990), 333-353. MR 1035413 Zbl 0702.58077

[Donnelly and Fefferman 1990b] H. Donnelly and C. Fefferman, "Nodal sets of eigenfunctions: Riemannian manifolds with boundary”, pp. 251-262 in Analysis, et cetera, edited by P. H. Rabinowitz and E. Zehnder, Academic Press, Boston, 1990. MR 1039348 Zbl 0697.58055

[Girouard and Polterovich 2014] A. Girouard and I. Polterovich, "Spectral geometry of the Steklov problem", preprint, 2014. arXiv 1411.6567

[Han and Lin 2008] Q. Han and F.-H. Lin, "Nodal sets of solutions of elliptic differential equations", book in preparation, 2008, available at http://www.nd.edu/qhan/nodal.pdf.

[Hardt and Simon 1989] R. Hardt and L. Simon, "Nodal sets for solutions of elliptic equations", J. Differential Geom. 30:2 (1989), 505-522. MR 1010169 Zbl 0692.35005

[Hezari and Sogge 2012] H. Hezari and C. D. Sogge, "A natural lower bound for the size of nodal sets", Anal. PDE 5:5 (2012), 1133-1137. MR 3022851 Zbl 1329.35224

[Jost 1984] J. Jost, Harmonic maps between surfaces, Lecture Notes in Mathematics 1062, Springer, Berlin, 1984. MR 754769 Zbl 0542.58002

[Lin 1991] F.-H. Lin, "Nodal sets of solutions of elliptic and parabolic equations", Comm. Pure Appl. Math. 44:3 (1991), 287-308. MR 1090434 Zbl 0734.58045

[Polterovich et al. 2015] I. Polterovich, D. A. Sher, and J. A. Toth, "Nodal length of Steklov eigenfunctions on real-analytic Riemannian surfaces", preprint, 2015. arXiv 1506.07600

[Sogge and Zelditch 2011] C. D. Sogge and S. Zelditch, "Lower bounds on the Hausdorff measure of nodal sets", Math. Res. Lett. 18:1 (2011), 25-37. MR 2770580 Zbl 1242.58017

[Sogge and Zelditch 2012] C. D. Sogge and S. Zelditch, "Lower bounds on the Hausdorff measure of nodal sets, II", Math. Res. Lett. 19:6 (2012), 1361-1364. MR 3091613 Zbl 1283.58020

[Sogge et al. 2015] C. D. Sogge, X. Wang, and J. Zhu, "Lower bounds for interior nodal sets of Steklov eigenfunctions", preprint, 2015. To appear in Proc. Amer. Math. Soc. arXiv 1503.01091

[Wang and Zhu 2015] X. Wang and J. Zhu, "A lower bound for the nodal sets of Steklov eigenfunctions", Math. Res. Lett. 22:4 (2015), 1243-1253. MR 3391885

[Zelditch 2008] S. Zelditch, "Local and global analysis of eigenfunctions on Riemannian manifolds", pp. 545-658 in Handbook of geometric analysis, I, edited by L. Ji et al., Adv. Lect. Math. 7, International Press, 2008. MR 2483375 Zbl 1176.58017 
[Zelditch 2014] S. Zelditch, "Measure of nodal sets of analytic Steklov eigenfunctions", preprint, 2014. arXiv 1403.0647 [Zhu 2015] J. Zhu, "Doubling property and vanishing order of Steklov eigenfunctions", Comm. Partial Differential Equations 40:8 (2015), 1498-1520. MR 3355501 Zbl 1323.47017

Received 8 Jul 2015. Revised 20 Dec 2015. Accepted 26 Feb 2016.

JIUYI ZHU: jzhu43@math . jhu .edu

Department of Mathematics, Johns Hopkins University, 313 Krieger Hall, 3400 N. Charles Street, Baltimore, MD 21218, United States 


\title{
Analysis \& PDE
}

msp.org/apde

\section{EDITORS}

\author{
EDITOR-IN-CHIEF \\ Patrick Gérard \\ patrick.gerard@math.u-psud.fr \\ Université Paris Sud XI \\ Orsay, France
}

BOARD OF EDITORS

Nicolas Burq Université Paris-Sud 11, France nicolas.burq@math.u-psud.fr

Massimiliano Berti Scuola Intern. Sup. di Studi Avanzati, Italy berti@sissa.it

Sun-Yung Alice Chang Princeton University, USA chang@math.princeton.edu

Michael Christ University of California, Berkeley, USA mchrist@math.berkeley.edu

Charles Fefferman Princeton University, USA cf@math.princeton.edu

Ursula Hamenstaedt Universität Bonn, Germany ursula@math.uni-bonn.de

Vaughan Jones U.C. Berkeley \& Vanderbilt University vaughan.f.jones@vanderbilt.edu

Vadim Kaloshin University of Maryland, USA vadim.kaloshin@gmail.com

Herbert Koch Universität Bonn, Germany koch@math.uni-bonn.de

Izabella Laba University of British Columbia, Canada ilaba@math.ubc.ca

Gilles Lebeau Université de Nice Sophia Antipolis, France lebeau@unice.fr

László Lempert Purdue University, USA lempert@math.purdue.edu

Richard B. Melrose Massachussets Inst. of Tech., USA rbm@math.mit.edu

Frank Merle Université de Cergy-Pontoise, France Frank.Merle@u-cergy.fr

William Minicozzi II Johns Hopkins University, USA minicozz@math.jhu.edu

Clément Mouhot Cambridge University, UK c.mouhot@dpmms.cam.ac.uk
Werner Müller

Yuval Peres

Gilles Pisier

Tristan Rivière

Igor Rodnianski

Wilhelm Schlag

Sylvia Serfaty

Yum-Tong Siu

Terence Tao

Michael E. Taylor

Gunther Uhlmann

András Vasy

Dan Virgil Voiculescu

Steven Zelditch

Maciej Zworski
Universität Bonn, Germany mueller@math.uni-bonn.de

University of California, Berkeley, USA peres@stat.berkeley.edu

Texas A\&M University, and Paris 6 pisier@math.tamu.edu

ETH, Switzerland riviere@math.ethz.ch Princeton University, USA irod@math.princeton.edu University of Chicago, USA schlag@math.uchicago.edu New York University, USA serfaty@cims.nyu.edu Harvard University, USA siu@math.harvard.edu University of California, Los Angeles, USA tao@math.ucla.edu Univ. of North Carolina, Chapel Hill, USA met@math.unc.edu

University of Washington, USA gunther@math.washington.edu

Stanford University, USA andras@math.stanford.edu

University of California, Berkeley, USA dvv@math.berkeley.edu

Northwestern University, USA zelditch@math.northwestern.edu

University of California, Berkeley, USA zworski@math.berkeley.edu

PRODUCTION

production@msp.org

Silvio Levy, Scientific Editor

See inside back cover or msp.org/apde for submission instructions.

The subscription price for 2016 is US \$235/year for the electronic version, and \$430/year (+\$55, if shipping outside the US) for print and electronic. Subscriptions, requests for back issues from the last three years and changes of subscribers address should be sent to MSP.

Analysis \& PDE (ISSN 1948-206X electronic, 2157-5045 printed) at Mathematical Sciences Publishers, 798 Evans Hall \#3840, c/o University of California, Berkeley, CA 94720-3840, is published continuously online. Periodical rate postage paid at Berkeley, CA 94704, and additional mailing offices.

APDE peer review and production are managed by EditFlow ${ }^{\circledR}$ from MSP.

PUBLISHED BY

- mathematical sciences publishers

nonprofit scientific publishing

http://msp.org/

(C) 2016 Mathematical Sciences Publishers 


\section{ANALYSIS \& PDE \\ Volume $9 \quad$ No. $4 \quad 2016$}

Peierls substitution for magnetic Bloch bands

Silvia Freund and Stefan TEUfEL

Dispersive estimates in $\mathbb{R}^{3}$ with threshold eigenstates and resonances

MARIUS BECEANU

Interior nodal sets of Steklov eigenfunctions on surfaces

859

JIUYI ZHU

Some counterexamples to Sobolev regularity for degenerate Monge-Ampère equations

881 CONNOR MOONEY

Mean ergodic theorem for amenable discrete quantum groups and a Wiener-type theorem for

893 compact metrizable groups

HUICHI HUANG

Resonance free regions for nontrapping manifolds with cusps

KIRIL DATCHEV

Characterizing regularity of domains via the Riesz transforms on their boundaries

Dorina Mitrea, Marius Mitrea and JoAn VERdERA 